\title{
DISRUPTIONS, INTERRUPTIONS AND INFORMATION ATTACK: IMPACT ON SITUATION AWARENESS AND DECISION MAKING
}

\author{
Mica R. Endsley \\ Debra G. Jones \\ SA Technologies, Inc.
}

\begin{abstract}
This paper presents a model of depicting the ways in which disruptions, interruptions and information attack can effect situation awareness and decision making in a variety of contexts. Those effected in military aviation include not just those in the cockpit, but also forward air controllers, ground based air traffic controllers and those in intelligence and support functions. The model incorporates the ways in which information attacks can effectively disrupt human decision making at various points in information processing. By carefully examining not just what cues might depict an attack to information systems, but also how human observers will be effected by such cues, more robust systems for protecting against disruptions and information attack can be developed.
\end{abstract}

\section{INTRODUCTION}

In the Information Age of the $21^{\text {st }}$ Century, our businesses, governments and personal activities have become highly dependant on information systems. Reports of the antics of hackers have become common place in the media. The results of most of these attacks range from embarrassment to nuisance to serious economic losses. In aviation, the consequences of successful or undetected information attacks can be even more catastrophic.

We have recently been involved in an effort to better understand the effects of information attack from a cognitive perspective. How do decision makers perceive and process information attacks within the context of their ongoing activities? The answer to this question lies in considering not just how they perceive an information attack, but how that event looks within the context of everyday disruptions (e.g. software glitches, computer crashes, ordinary maintenance) that may look very similar. How people interpret and comprehend the cues they perceive is as important to understanding human cognition under information attack as are the systems that present the cues.

To this end we undertook an effort to develop a model of situation awareness (SA) and decision making that reflects the way in which these cognitive processes may be effected by normal interruptions, disruptions and covert information attack. This model was developed based on prior work on SA and decision making (Endsley, 1988; Endsley, 1995; Endsley and Jones, 1997; Klein, 1989), and on observations in settings established for detecting information attacks.

\section{MODEL OF THE EFFECT OF DISRUPTIONS ON SA AND DECISION MAKING}

In order to depict the effects of disruptions and information attack, on SA and decision making, it is important to understand the types of decisions that people in a position to detect attacks are making and the processes they use in order to make those decisions. This is depicted in Figure 1.

Such decisions are not discrete, made at one point in time, but rather must be made within the context of a background of noisy information: system failures and glitches, system maintenance, normal user problems (e.g. forgotten passwords), and similar problems that are part and parcel of today's technology. Any data or information perceived is examined within this context. The decision-maker must determine whether the cues represent something abnormal, or are part of a known class of "typical" problems that exist within daily operations. Very often, if the cues fit a typical pattern or can be explained away to fit a known ongoing situation (e.g. system maintenance), then the possibility of an abnormal event, such as a hostile attack, may never be entertained. Situation awareness requirements in this domain are those that allow the decision-maker to address the types of questions shown in Figure 1.

Following this backdrop, and in consideration of models of naturalistic decision making (Klein,1989) and situation awareness (Endsley, 1988, 1995), a model is proposed to explain the effects that disruptions can have on situation awareness and decision making. The model proposed is a fairly broad one, and includes interruptions and information disruptions that may not be hostile in 
origin, but which may affect SA and decision making in very similar ways. Disruptions are categorized into four major categories:

(1) Disruptions that affect information preprocessing,
(2) Disruptions that affect prioritization and attention,

(3) Disruptions that affect confidence in information, and

(4) Disruptions that affect interpretation.

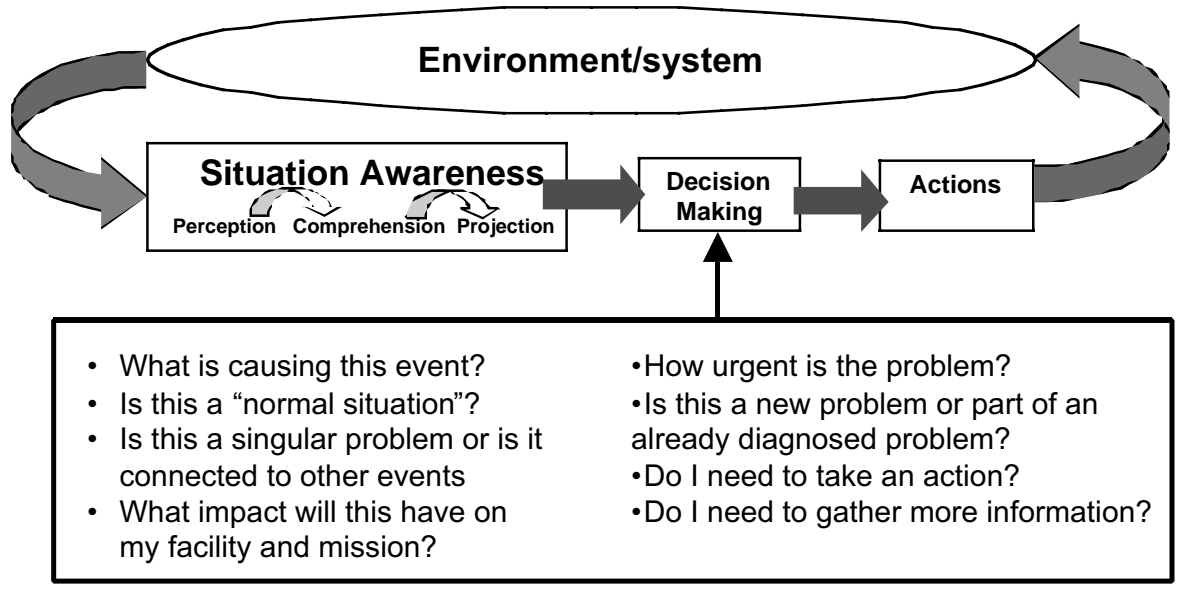

Figure 1. Decision Context for Detecting and Diagnosing Information Attacks

\section{Disruptions in Information Pre-Processing}

Disruptions that effect information pre-processing can arise from a number of factors: the production of too much information or too fast a flow of information, disorganized information content, dissonant information (where information from different sources disagree) and delayed information, as shown in Figure 2. These factors are common in the cockpit, in air traffic control and in many aspects of aviation.

These problems or disruptions will likely affect the pre-processing of information as the decision maker attempts to find needed information, sort through what is available and integrate it with known information to form ongoing situation awareness. These disruptions can have the effect of causing the decision maker to omit key information, thus leading to an incorrect picture of the situation, and can dramatically slow information processing thus leaving far less time available for decision making.

It is worth noting that these factors may be naturally occurring within the environment (benign) or due to malicious attacks. Since these factors are a "normal" part of many systems, the use in an information attack, or the cue they provide that one is under information attack, may be very difficult to detect. Thus if an information attack is disguised as an information overflow, it may easily be misinterpreted as due to normally occurring benign causes and will likely slow the effective search for a cause and cure. Relevant cues will be lost in the maelstrom. Similarly, one major cue of an information attack may be the presence of dissonant information. Yet, the disagreement of information from different sources may be very common due to differences in technologies, and thus an attack may not be recognized as such. Information dissonance is likely to lead to certain information being ignored (discounted), and will slow decision-making.

\section{Disruptions in Prioritization and Attention}

Disruptions that effect the ability of the decisionmaker to prioritize and direct attention effectively are shown in Figure 3. Task interruptions are a key form of this type of disruptions. Interruptions to the flow of information processing (e.g. new competing tasks) are a major source of SA problems. Interruptions (by ATC or other cockpit alarms, signals or events) are common in the cockpit, and have been traced to numerous accidents.

On an ongoing basis decision-makers must juggle multiple goals and process environmental information to help insure that these goals and tasks are correctly prioritized. The prioritization of goals and tasks is key to determining how the person will direct their attention and interpret information perceived. This takes the form of alternating top-down (goal-driven) processing and 


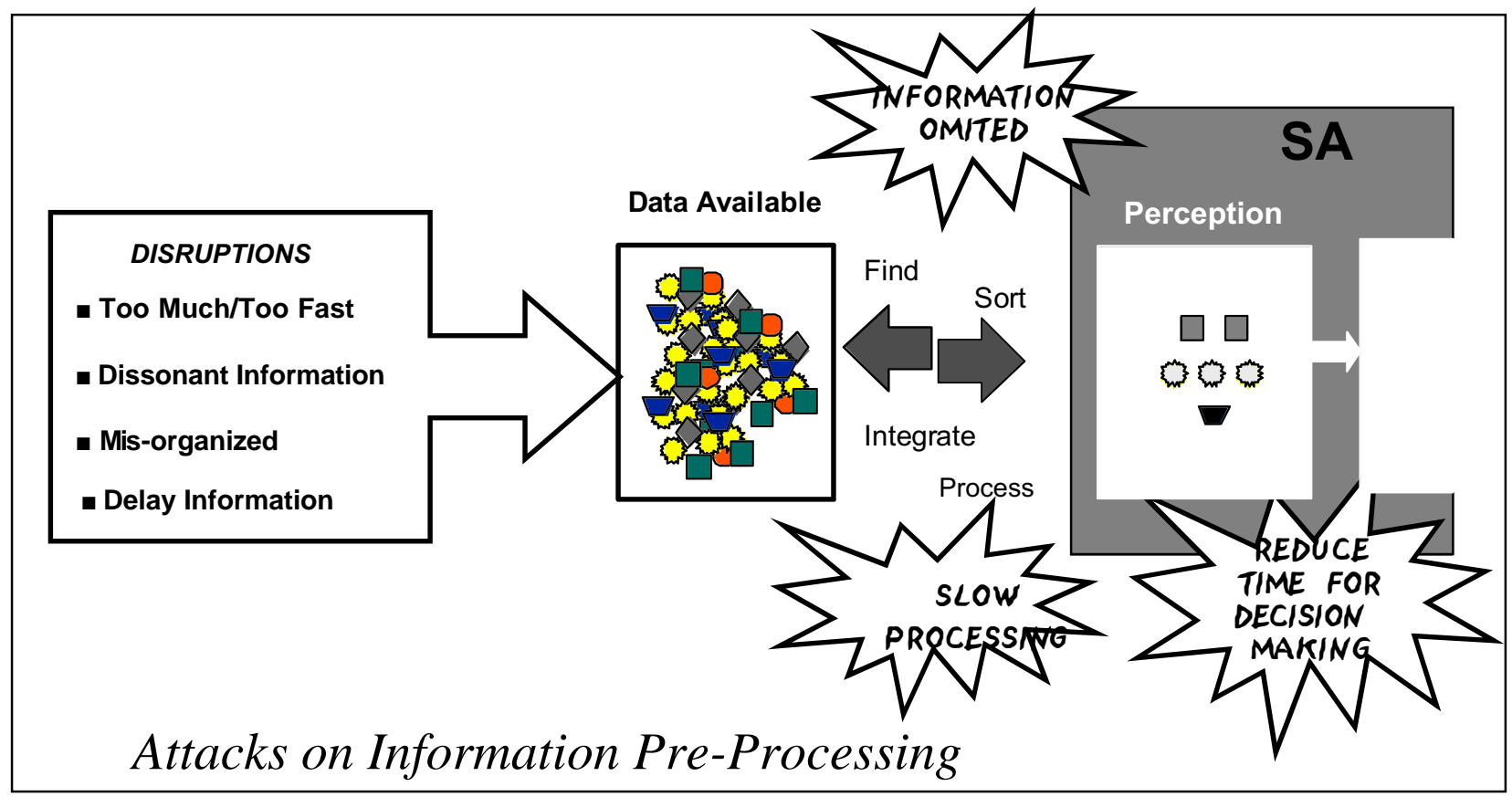

Figure 2: Disruptions in SA: Pre-processing of Information

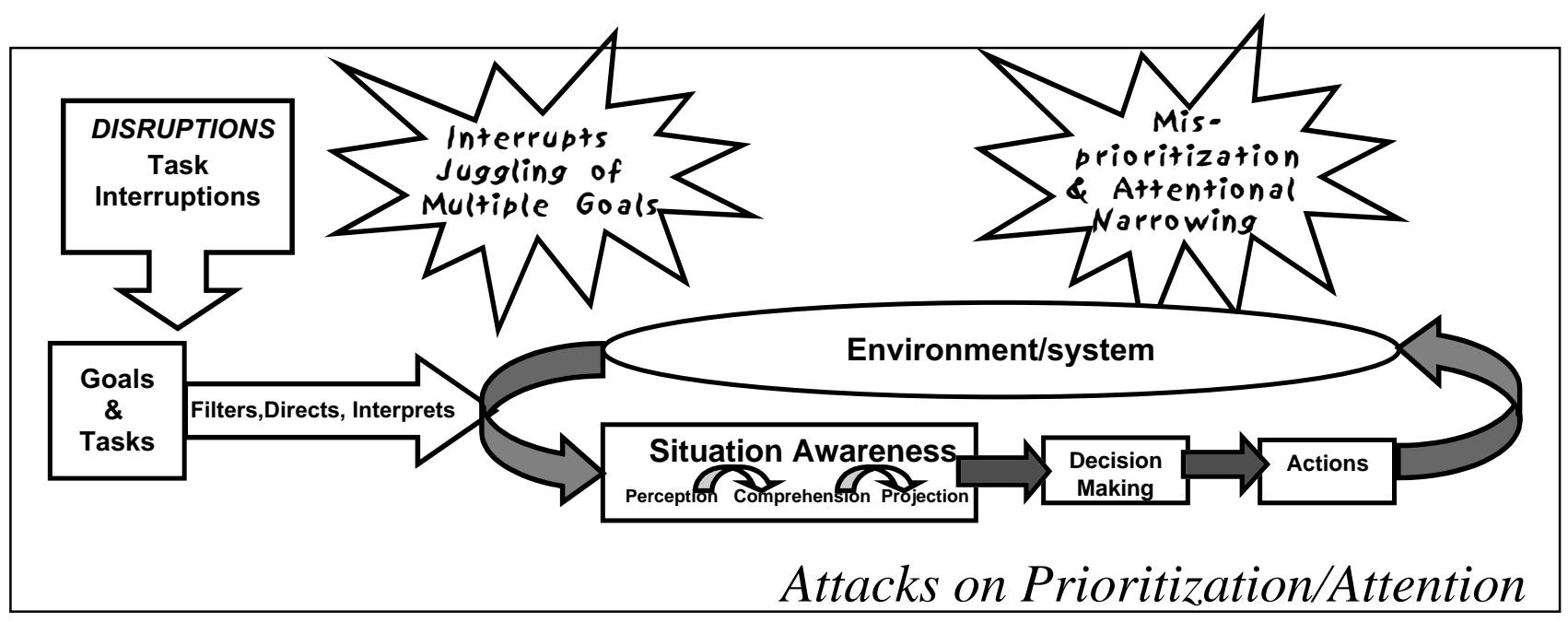

Figure 3: Disruptions in SA: Prioritization and Attention

bottom-up (data driven) processing. Interruptions can be very disruptive to this cycle. A common failure will be for people to focus in on the interrupting task and forget to manage other competing goals and tasks (attentional narrowing). People may also be poor at prioritizing the interruption in relation to othergoals, as they have lost sight of those competing goals and tasks from short-term memory. Either of these problems can lead to critical losses of SA and poor decision-making (failure to recognize the information attack). Because interruptions are frequent in aviation, their use in information attack may go undetected, but it is possible to harness this effect for nefarious purposes.

\section{Disruptions in Information Confidence Level}

Another form of information attack may be found in attacks that effect the decision maker's confidence in 
certain information, shown in Figure 4. The confidence level of information received has been found to be an important part of SA in analyses of SA requirements in commercial and military aviation as well as ATC and many other domains. Such attacks may partially corrupt some information or may lead the decision-maker to believe that a certain information source is unreliable (compromised or faulty). Even if the information perceived is true and correct, the fact that the decision- maker has lower confidence in the information can lead to negative effects on SA and decision making. They will be less likely to act on information considered unreliable and will be more likely to spend more time seeking more information to confirm or deny it. Thus the decision maker is likely to be ineffective, even with good information about the situation. Attacks on a decision-maker's confidence in information are therefore particularly sneaky, and may be very difficult to detect.

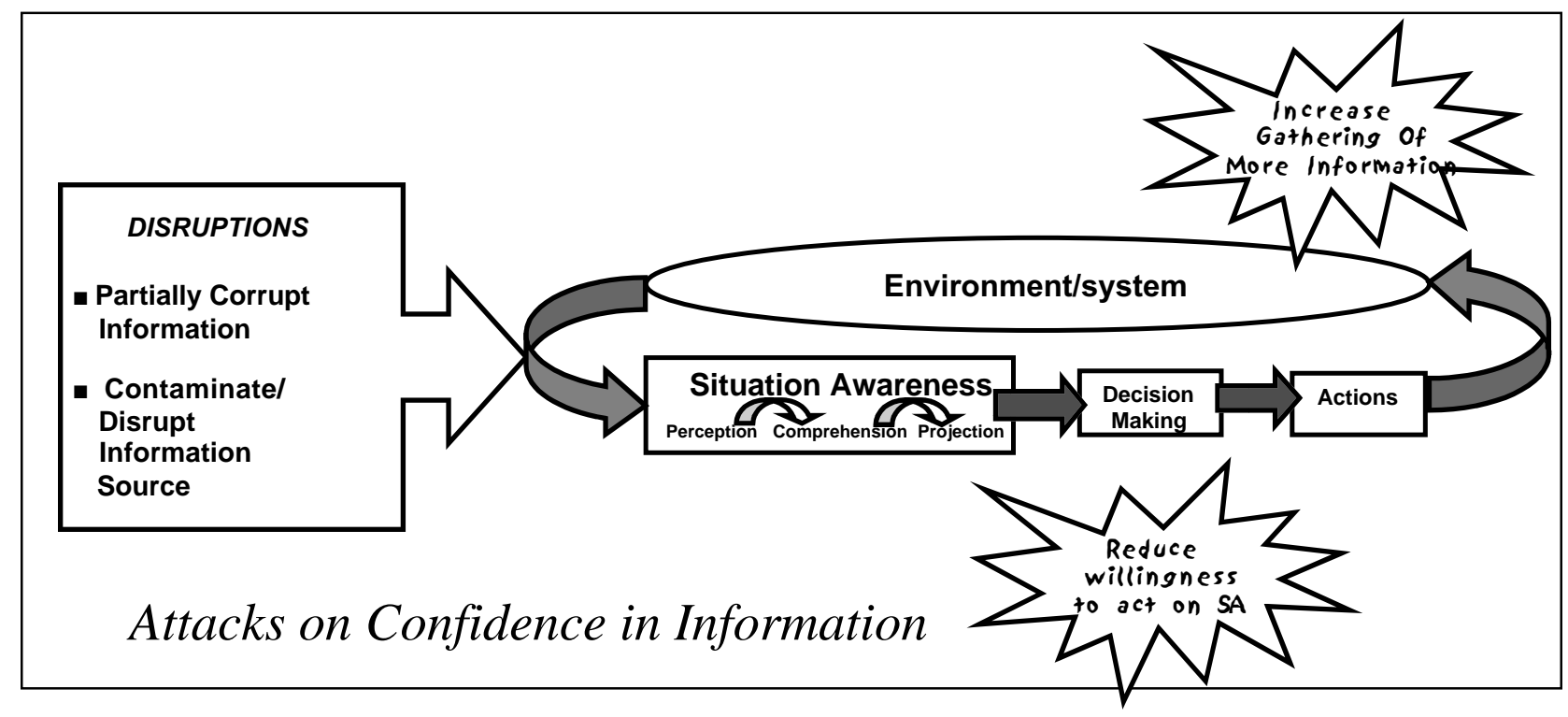

Figure 4: Disruptions in SA: Confidence in Information

\section{Disruptions in Information Interpretation}

Finally, disruptions that can effect SA and decision making may be directed at effecting the way in which perceived information is interpreted, Figure 5. These disruptions may include the deliberate insertion of cues or mimicking of cues that would be consistent with known "normal" situations (e.g. maintenance, a user forgetting a password), for instance. As the decisionmaker may pattern match between detected environmental cues and known classes of situations in memory, such a factor could well lead to the person matching cues to "normal situations" and thus misinterpreting cues to the contrary. This type of attack is quite difficult to overcome, as once a particular mental model has been triggered, it may be quite difficult for cues indicating a different type of situation to trigger the discovery of the error (Jones, 1997). They will most likely be explained away to fit the mental model that is active.

Other forms of attack that may lead to this type of disruption also include withholding critical cues that would indicate a different "non-normal" class of situations and creating dissonant information that may also lead to pattern matching to the wrong class of situations. Once the wrong situation model is activated, it may be quite difficult to detect cues that they are really under attack.

\section{Summary}

This model depicts the effects that various types of disruptions or interruptions that may occur naturally in aviation and other domains can have on a $n$ individual's SA and decision making. In addition, this model provides a basis for analyzing the way in which nonnormal information attacks may be interpreted within a normal context. Such attacks should be considered by military aviators and decision makers, and may become more common place in commercial aviation and industry as terrorist activities increase. This model of the effect of disruptions on SA and decision making is preliminary and will be further developed and expanded through additional observation and testing in the aviation environment where such disruptions are common. The intention of the model is to help direct efforts at creating 
systems for supporting decision makers in effectively comprehending and dealing with information attacks and normal disruptions. It is being used to develop decision support tools for detecting such attacks within the context of normal disruptions and interruptions.

\section{ACKNOWLEDGEMENTS}

This work was developed under an SBIR to the U.S. Air Force Research Laboratory, under subcontract to Aptima, Inc.. We'd like to thank the support of.Gil Kuperman, COTR, and our colleagues at Aptima, Daniel Serfaty and John Poirier, and at Klein Associates, Gary Klein and Michael McCloskey.

\section{REFERENCES}

Endsley, M. R. (1988). Design and evaluation for situation awareness enhancement. Proceedings of the
Human Factors Society 32nd Annual Meeting (pp. 97-101). Santa Monica, CA: Human Factors Society.

Endsley, M. R. (1995). Toward a theory of situation awareness in dynamic systems. Human Factors, 37(1), 32-64.

Endsley, M. R., and Jones, W. M. (1997). Situation awareness, information dominance, and information warfare (AL/CF-TR-1997-0156). Wright-Patterson AFB, OH: United States Air Force Armstrong Laboratory.

Jones, D. G. (1997). Reducing situation awareness errors in air traffic control. Proceedings of the Human Factors and Ergonomics Society 41st Annual Meeting (pp. 230-233). Santa Monica, CA: Human Factors and Ergonomics Society.

Klein, G. A. (1989). Recognition-primed decisions. In W. B. Rouse (Eds.), Advances in man-machine systems research (pp. 47-92). Greenwich, Conn: JAI Press, Inc.

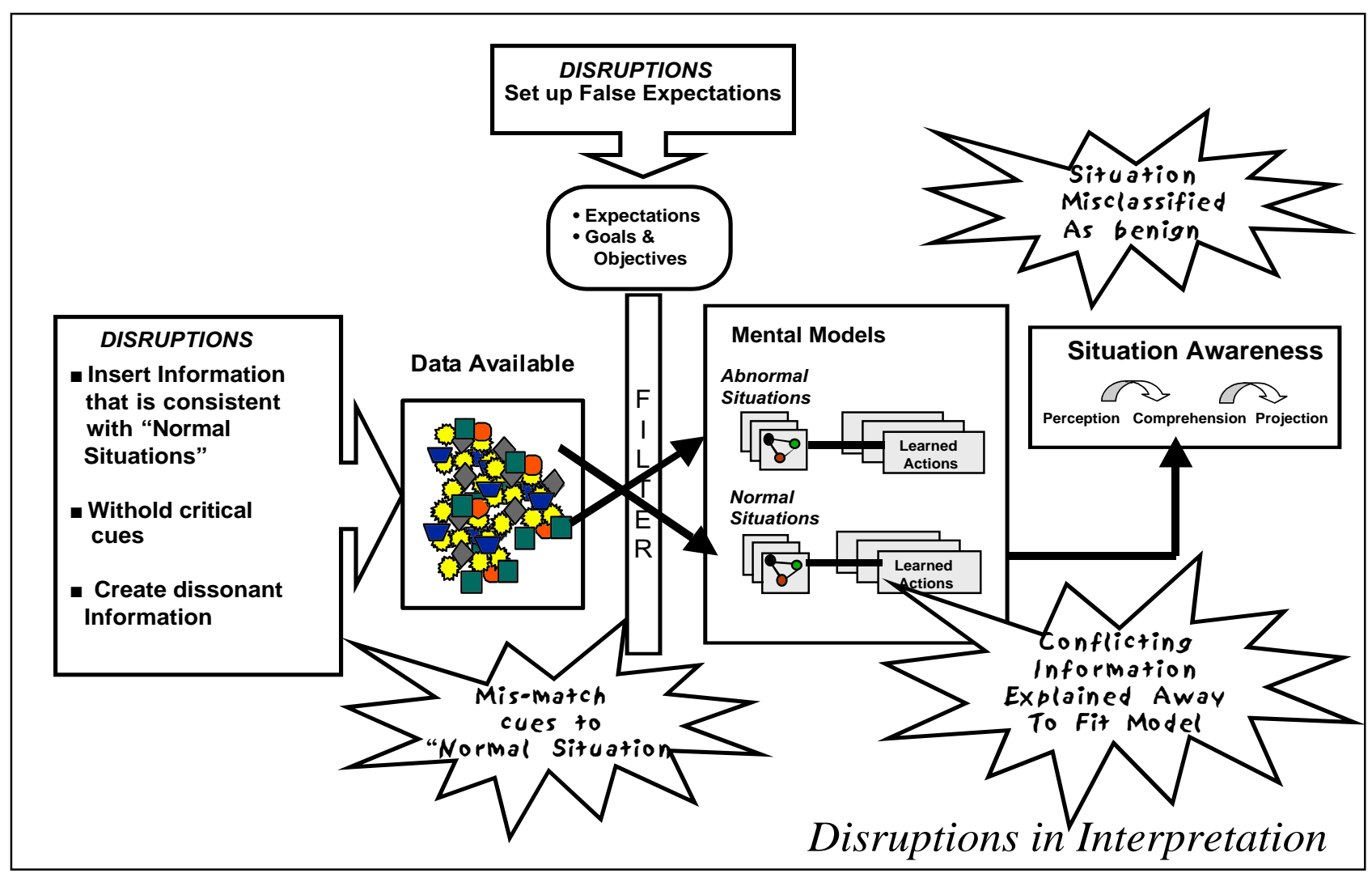

Figure 5: Disruptions in SA: Interpretation of Information 